\title{
Risk factors of extended-spectrum beta-lactamase- producing Enterobacteriaceae bacteremia in Thai emergency department: a retrospective case-control study
}

\author{
Khrongwong Musikatavorn ${ }^{\mathrm{a}}$, Chatgaew Chumpengpan ${ }^{\mathrm{b}}$, Chaita Sujinpram ${ }^{\mathrm{b}}$ \\ ${ }^{a}$ Emergency Medicine Unit, Department of Medicine, Faculty of Medicine, Chulalongkorn University, \\ Bangkok 10330; ${ }^{b}$ Emergency Medicine Unit, Outpatient Department, King Chulalongkorn Memorial \\ Hospital, Bangkok 10330, Thailand
}

\begin{abstract}
Background: Infections caused by resistant extended-spectrum beta-lactamase (ESBL)-producing enteric bacteria and their risk factors are globally recognized. However, such risk factors have not been explored in emergency department (ED) where the first choice of empirical antimicrobials is crucial.

Objective: Determine risk factors of ESBL bacteremia in ED, especially in our geographic area.

Methods: A retrospective case-double-control study was conducted at King Chulalongkorn Memorial Hospital. All adult ED patients with ESBL-producing E. coli and K. pneumoniae in blood cultures between October 2007 and October 2008 were recruited for this study. The potential risk factors were analyzed and compared with nonESBL-producing bacteremic patients (control group 1) and matched general ED patients (control group 2). Nonbeta-lactam susceptibility testing among the cohort was also evaluated.

Results: Thirty ESBL (cases), 103 group 1 controls, and 100 group 2 controls were assessed. Based on the univariate analysis, age, chronic kidney diseases, malignancy, poor functional status, previous hospitalization within 90 days, and previous antimicrobial exposure especially to cephalosporins, quinolones, and carbapenems within 30 days were the risk factors for ESBL bacteremia compared with both types of control patients. Age $\geq 60$ and previous cephalosporin use were consistently identified as the risk factors by multivariate models using both control groups. The susceptibility to non-beta-lactam agents in the ESBL group was significantly lower than the non-ESBL. No carbapenem resistance was found.

Conclusion: Elderly ED patients, especially those who had previous cephalosporin exposure within 30 days, were at higher risk of ESBL-producing bacteremic infections. ESBLs tended to have less susceptibility to the non- $\beta$ lactam agents.
\end{abstract}

Keywords: Bacteremia, emergency department, extended-spectrum beta-lactamase, risk factors

The problem of antimicrobial resistance is now globally recognized in both gram-negative and grampositive infections, especially in inpatient and intensive care unit (ICU) settings. Emergency Department (ED) patients and outpatients, contributing most of the community-acquired infections, are also of concern. In the ED septic patients, the antimicrobial

Correspondence to: Khrongwong Musikatavorn, MD. Emergency Medicine Unit, Department of Medicine, Faculty of Medicine, Chulalongkorn University, Bangkok 10330, Thailand.E-mail:kmusikatavorn@yahoo.com agents are required as soon as possible since delay response can increase the mortality rate $[1,2]$. In such a time-dependent clinical management of septic patients in ED, recognition of the antimicrobial resistance burden can help clinicians make the decisions to use the antimicrobial properly to control the infections while minimizing further antimicrobial resistance by giving "too broad" spectrum agents.

Infections with enterobacteria were prevalent in community-acquired infections. Yet, community acquisition of antimicrobial resistance in gram-negative pathogens is not uncommon throughout the United 
States, Europe [3-6], and certain regions of Asia $[7,8]$.

In Thailand, the patterns of antimicrobial resistance may be different from other regions. In fact, Apisarnthanarak et al. [9] pointed out this growing problem. No previous comparable study focusing solely on ED patients has been published. Unpublished data of common gram-negative (Escherichia coli, Klebsiella pneumoniae and Salmonella spp.) antimicrobial resistance obtained in our ED showed an increasing trend over the past five years, implying that modifying factors were being exposed to our ED patients and making these at risk of infections by resistant strains.

In this study, we investigated risk factors of extended spectrum beta-lactamase (ESBL)-producing bacteremia among the patients with enterobacterial infection. This may improve the clinical decision of prompt antimicrobial administration in those who are infected with enterobacteria in ED because infection with resistant gram-negative pathogens due to inadequate antimicrobial therapy has been associated with poor clinical and survival outcomes [10-13].

\section{Methods}

The retrospective case-double-control study was conducted at King Chulalongkorn Memorial Hospital $(\mathrm{KCMH})$. The study was approved by Ethics Committee of KCMH. No written informed consent was required.

We retrospectively collected data from ED patients (age: older than 15 years) that attended between October 2007 and October 2008 and whose blood culture specimens yielded E. coli and K. pneumoniae from the hospital laboratory computer database. We collected demographic data, co-morbidities, baseline performance status (using Karnofsky performance status, KPS, scores), sources of infection, previous antimicrobial use, and other potential risk factors of the patients. To provide accurate information, all outpatient medical records were reviewed in the following three steps, 1) manual working though the paper records, 2) electronic medical prescription database, and 3) medical reconciliation data, if any. None of the specimens was duplicated.

When bacteremic patients were identified, we categorized those with ESBL-producing organisms in their blood cultures and those with non-ESBLproducing strains into cases and susceptible-form controls (control group 1), respectively.
Matched population-based controls were used in comparison between the cases. In this group, control patients were recruited from patients admitted to the wards from the same ED according to other conditions on the same day when the eligible specimens of the case patients were collected. They were not of E. coli and K. pneumoniae bloodstream infection at the time of admission. These matched controls were randomly selected proportionate to a case by $3: 1$ ratio and termed as control group 2 .

\section{Determining the sites of infection}

Concomitant sites of infection were determined as follows [14-17].

- Urinary tract infections: fever, flank pain, lower urinary tract symptoms (e.g., frequency, urgency, and dysuria) with pyuria (at least, 10 leukocytes $/ \mathrm{mm}^{3}$ ), or $\geq 10^{5}$ bacteria/mL in uncentrifuged urine (corresponding with the blood culture)

- Respiratory tract infections: pneumonias (fever, cough, dyspnea, and new infiltration on chest radiograph) and their complications

- Hepatobiliary tract infections: acute cholecystitis (fever, right upper quadrant pain, and ultrasonographic findings of inflamed gall bladder) or acute cholangitis (fever, right upper quadrant pain with or without jaundice, biochemical and radiographic evidences of obstructed bile duct or presence of structural abnormalities, or foreign materials e.g. postsphincterotomy or stents)

- Skin and soft tissue infections: fever with skin and subcutaneous tissue inflammation (cellulitis), including deeper fascial infection (necrotizing fasciitis) and other gangrenous cellulitis

- Primary bacteremia: the presence of bacteria in the blood without a definite or clinically evident concurrent source of infection

\section{Microbiological methods and antimicrobial sensitivity testing}

In vitro antimicrobial susceptibility testing, minimal inhibitory concentration (MIC), and the cutoff points for susceptible (S), intermediately resistant (I), or resistant $(\mathrm{R})$ organisms were done and determined by disc diffusion method (Becton Dickinson ${ }^{\circledR}$, NJ, USA and Oxoid ${ }^{\circledR}$, Cambridge, UK) and E-test (AB biodisk ${ }^{\circledR}$, Solna, Sweden). For enterobacteria (e.g. E.coli and K.pneumoniae) that were growing in blood cultures, susceptibility testing was performed using the following lists of antimicrobials: ampicillin, cefazolin, 
trimetroprim/sulfamethoxazole, amoxicillin/clavulonic, gentamicin, amikacin, ciprofloxacin, cefoperazone/ sulbactam, piperacillin/tazobactam, cefoxitin, cefotaxime, ceftriaxone, ceftazidime, imipenem, meropenem, and ertapenem.

We routinely did ESBL testing in $K$. pneumoniae, $K$. oxytoca, E. coli, and bacteremic P. mirabilis, according to the Clinical and Laboratory Standards Institute (CLSI) manual [18]. The ESBL-producing organisms were phenotypically identified by the double disk or combined disk diffusion methods (Becton Dickinson $^{\circledR}$, NJ, USA), and E-test (AB biodisk ${ }^{\circledR}$, Solna, Sweden)

All protocols, inhibition zone diameters and MIC breakpoints were followed in accordance with the CLSI manual [18].

\section{Statistical analysis}

Descriptive statistical analyses were used for demographic data in terms of percentages between the two groups of patients. Univariate chi-square $\left(\chi^{2}\right)$ and multivariate logistic regression analyses were performed to explore the potential risk factors for ESBL bacteremia. Odds ratio (OR) and 95\% confidence intervals $(95 \% \mathrm{CI})$ were demonstrated. Exploratory backward method was used in our multivariate analysis. The factors in the univariate models were chosen for entering the multivariate analysis if the setting of p-value was 0.15 , and backward elimination method with $p$-value $=0.05$ to remove the variables from the model [19]. Normallydistributed continuous variable was analyzed using student's t-test where appropriate.

The resistance rate was calculated as the number of resistant or intermediately-resistant strains divided by the total number of strains. The difference was considered statistically significant if $p$-values were $<0.05$. All statistical analyses were performed by Statistical Package for the Social Sciences (SPSS Inc. Chicago, USA) version 16.0.

\section{Results}

Ninety-seven and 38 blood culture specimens grew E. coli and K. pneumoniae, respectively. One hundred thirty five samples were analyzed, of which two were missing ESBL phenotype. Thus, 133 patients were identified and considered eligible. These included 103 in the non-ESBL group (control group 1) and 30 in the ESBL group (cases). The rate of ESBLproducing $E$. coli and $K$. pneumoniae bacteremia among the bacteremic strains were $21.6 \%$ (21/97) and $23.7 \%(9 / 38)$, respectively.

Concerning the control group 2, one hundred matched controls were randomly chosen to identify the potential factors of the ESBL-producing bacteremic cases since 30 cases were discovered during the study period.

The demographic data and patient characteristics of the cases and both control groups are summarized in Table 1.

Comparing between the cases and control group $1,52.6 \%$ of the patients $(70 / 133)$ had a history of some hospitalization within 90 days or antimicrobial exposure within 30 days. Based on univariate analysis, mean age, chronic kidney diseases, malignancy, KPS scores $\leq 40$, previous hospitalization within 90 days, and previous antimicrobial use especially cephalosporins, quinolones, and carbapenems within 30 days were the risk factors for ESBL bacteremia. As shown in Table 1, the mean ages of the ESBL group were significantly older than that of the non-ESBL group, but there were marginally insignificant when categorized into age $\geq 60$. Multivariable analysis showed two significant independent factors of ESBL bacteremia, age $\geq 60$ and previous cephalosporin use. Comparing with matched control population (control group 2), age, chronic kidney diseases, malignancy, KPS score $\leq 40$, previous hospitalization within 90 days, and the use of cephalosporins, quinolones, and carbapenems within 30 days were the risk factors in univariate analysis. This is similar to the conditions in control group 1. Multivariate analysis demonstrated four independent risk factors, age $\geq 60$, chronic kidney diseases or malignancy, previous use of cephalosporins, and carbapenems within 30 days.

\section{Susceptibility test to various antimicrobials}

Susceptibility against other anti-gram-negative agents was analyzed in all eligible isolates. Table 2 shows a comparison between the ESBL- with the non-ESBL-producers. Interestingly, in addition to a resistance to $\beta$-lactams (penicillins and cephalosporins), the ESBL producers tended to be less susceptible to non- $\beta$-lactam antimicrobials than the non-ESBLs. No carbapenem resistance was found in either group. 


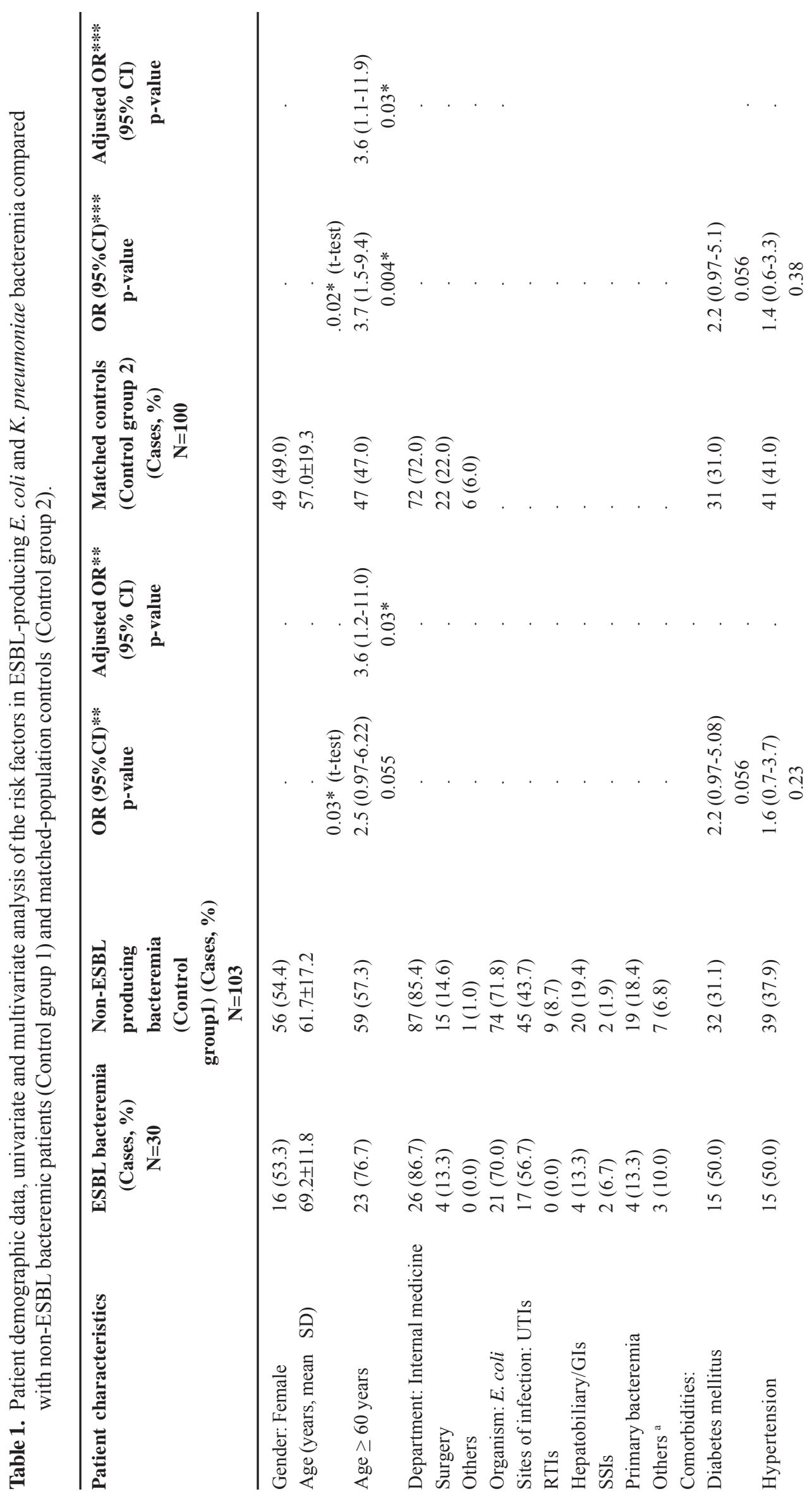




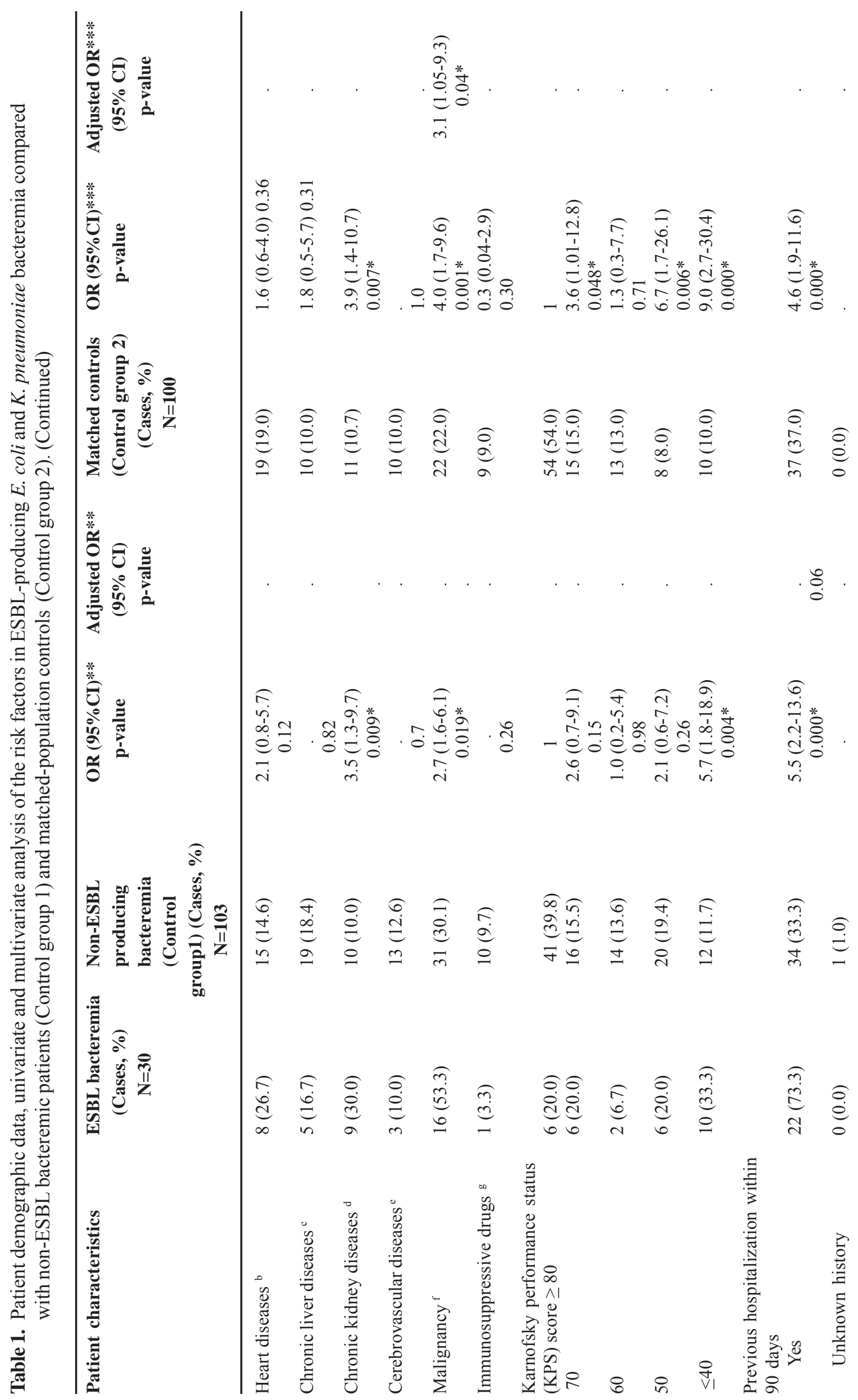




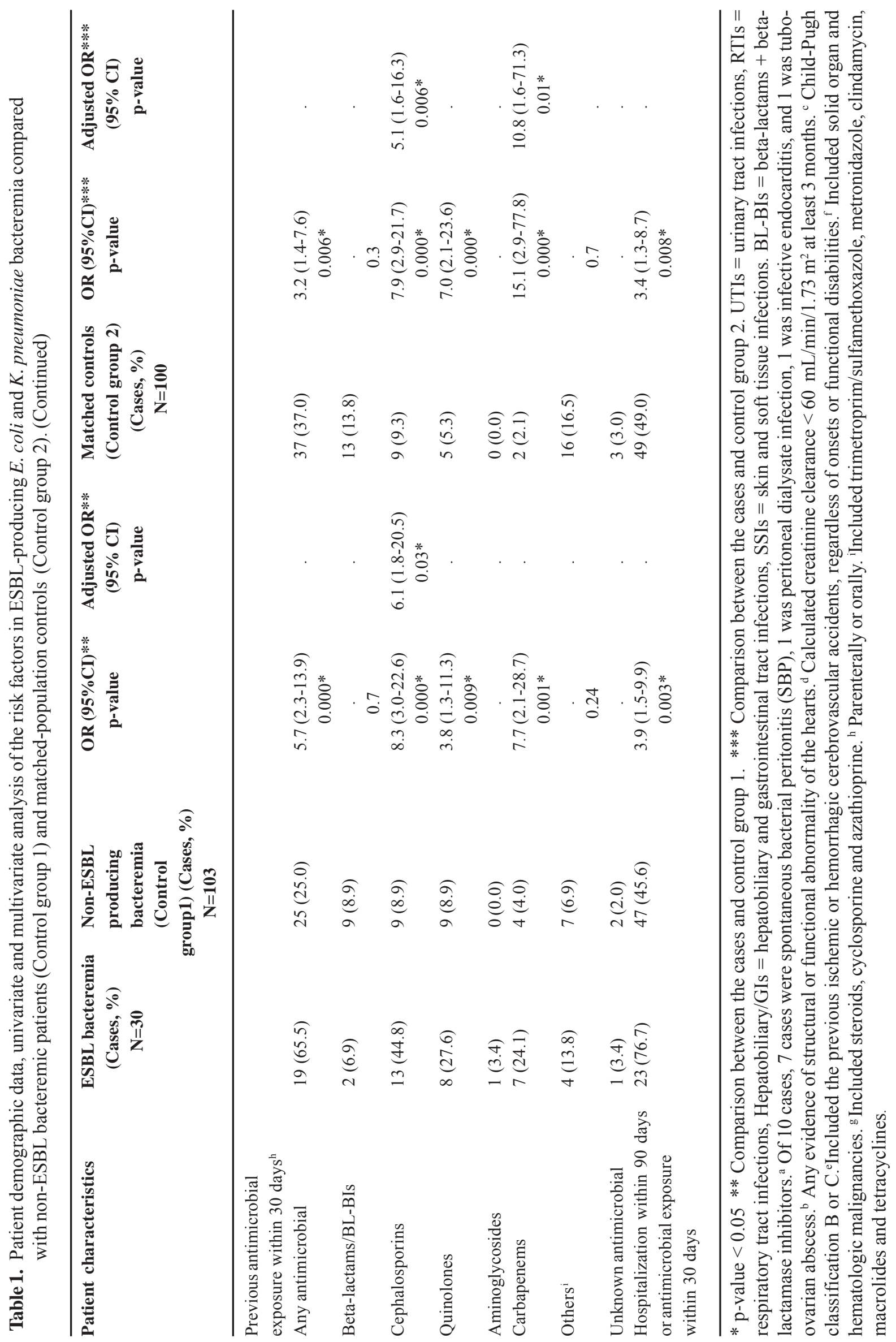


Table 2. In vitro susceptibility to various antimicrobials of non-ESBL and ESBL-producing E. coli and K. pneumoniae.

\begin{tabular}{lcccc}
\hline Antimicrobial agents & \multicolumn{2}{c}{ No. (\%) of susceptible isolates } & Odds ratio (95\%CI) & p-value \\
\cline { 2 - 3 } & $\begin{array}{c}\text { Non-ESBL } \\
\text { N=103 (100\%) }\end{array}$ & $\begin{array}{c}\text { ESBL } \\
\text { N=30 (100\%) }\end{array}$ & & \\
\hline Cefazolin & $92(90.2)$ & $0(0.0)$ & $4.0(2.3-6.8)$ & $0.000^{*}$ \\
Ceftriaxone/cefotaxime & $100(97.1)$ & $1(3.3)$ &. &. \\
Ceftazidime & $101(98.1)$ & $21(70.0)$ & $21.6(4.3-107.5)$ & $0.000^{*}$ \\
Cefepime & $103(100.0)$ & $20(66.7)$ &. & $\cdot$ \\
Ampicillin & $17(16.7)$ & $0(0.0)$ &. &. \\
Amoxicillin-clavulanicacid & $88(86.3)$ & $12(40.0)$ & $9.4(3.7-23.7)$ & $0.000^{*}$ \\
Ciprofloxacin & $76(73.8)$ & $12(40.0)$ & $4.2(1.8-9.9)$ & $0.001^{*}$ \\
Gentamicin & $86(83.5)$ & $18(60.0)$ & $3.3(1.4-8.3)$ & $0.006^{*}$ \\
Amikacin & $103(100.0)$ & $24(82.8)$ & & $\cdot$ \\
Carbapenems ** & $103(100.0)$ & $30(100.0)$ & &. \\
\hline
\end{tabular}

$*$ p-value $<0.05, * *$ Carbapenems $=$ imipenem, meropenem and ertapenem.

\section{Discussion}

The trend towards community-acquired antimicrobial resistance of enterobacteria, including ESBL, is well described worldwide. Yet, the rates are increasing drastically. The rates of ESBL-producing $E$. coli and $K$. pneumoniae among bacteremic patients in ED were approximately $20 \%$ according to our report. An article from another institute in our country reported that the overall prevalence of ESBL, of which most of them were hospital-acquired and included that of non-enterobacteria, was 30.1\% [20].This convinced us that the rates of ESBLproducing enterobacteria in Thailand were problematic.

Presentation of infections with enterobacteria had led the patients to the ED and the risk factors of ESBL infections among these were our main interest. Many studies have shown that ESBL bacteremia was one of the predictors associated with longer hospital stay [12] and higher mortality in the community [21], and hospital-acquired infections [22]. Age, poor functional status, certain co-morbidities such as chronic kidney diseases or malignancy, previous hospital hospitalization within 90 days, and previous antimicrobial exposure within 30 days were considered to be risk factors of ESBL enterobacterial bacteremia in both studies. In our multivariate analysis, older age group $(\geq 60)$ and previous cephalosporin exposure within 30 days were independent risk factors, while the latter was the strongest predictor in both studies using different type of controls. Furthermore, has been constantly demonstrated in previous publications though the intervals between the exposure and time of specimen collection varied among these (one to three months) $[3,11,23,24]$. Certain comorbidities such as chronic kidney diseases and malignancy were the independent predictors of ESBL bacteremia compared with general ED patients.

Previous carbapenem exposure within 30 days could be a factor resembling that of Martinez et al. [25], which mainly concerned in hospital-acquired infected patients. This demonstrated significance in our multivariate model only in general ED population-based study. The interaction between the cephalosporins and carbapenem exposure could be explained by the sequential use of carbapenems when clinically unresponsive to cephalosporins during the prior admission. Therefore, the influence of carbapenems was masked by the cephalosporins. About half of both control groups had a history of some hospitalization within 90 days or antimicrobial exposure within 30 days. As the patients were septic, they were defined as community-onset, healthcare-associated infections proposed by Friedman et al.[26]. It was also a factor according to the univariate models using both control groups. These findings could imply that healthcare-associated septic elderly patients in our ED, especially those who had certain co-morbidities and previous antimicrobial exposure, particularly cephalosporin or carbapenem, were at high risk of being infected with ESBL-producing pathogens when they were having enterobacterial infections. Such risk 
factors (age $>60$, health-care associated infection, and recent antimicrobial use) were comparable to the recent result by Rodr guez-Ba o et al. [27] that referred to community-onset ESBL-producing E.coli bacteremia at a multicenter level. However, previous cephalosporin and carbapenem exposure were considered the influential factors in our study.

Factors such as the use of antimicrobials may have some protective effect against yielding susceptible organisms that enter the "susceptibleform" control group [28]. Therefore, those could overestimate the OR of the risk factor [29, 30]. This might explain the exaggeration of the adjusted OR of previous cephalosporin exposure when comparing the cases with susceptible-form controls, thus, the reduction of the OR was observed in matched population-base control study. Our results confirmed that such risk factors were persistently germane to every sequence of the patient care, consistent with earlier publications. However, even if the adjusted predictors were obviously denoted, other clinically important variables, such as performance status, previous colonization with resistant strains, or overall clinical profiles should not be disregarded when being applied in clinical practice.

Quinolones, aminoglycosides or cephalosporins, frequently used against enterobacteria, were widely recommended in the past decade $[31,32]$. Our study revealed the ESBLs were also likely to have quinolone co-resistance since some of the patients had exposure to both cephalosporins and quinolones [33] or both resistances could be simultaneously adopted by plasmid-mediated mechanisms $[34,35]$. These agents can be insufficient for initial treatment if infections are ESBLs.

Antimicrobials must be promptly given to ED patients likely infected with ESBLs, especially those in a "cannot-wait" sepsis or septic shock state. Therefore, carbapenems may be considered early since no carbapenem resistance against either nonESBL-producing enterobacteria or the ESBL producers was found in our antimicrobial susceptibility report. Moreover, a publication has shown a better outcome if used in ESBL bacteremic cases [22].

\section{Conclusion}

ESBL-producing E. coli and K. pneumoniae were prevalent among the enterobacterial infection in our ED, similar to those in the hospitalized patients and other geographic areas. The risk factors of ESBL bacteremia were age, poor functional status, certain co-morbidities such chronic kidney diseases or malignancy, previous discharge from hospital, and previous antimicrobial exposure (cephalosporins, quinolones, and carbapenems). Old age and previous cephalosporin exposure were considered the most influential factors. ESBLs tended to have co-resistance to non- $\beta$-lactam antimicrobials. Emergency physicians should be aware of the high prevalence and the risks of ESBL infection when dealing with suspicious enterobacterial infections.

\section{Acknowledgement}

We would like to thank Wanla Kulwichit, MD (Department of Medicine, Chulalongkorn University) for his comments, Mr. Nirun Intarut (Clinical Research Center, Faculty of Medicine, Chulalongkorn University) for his suggestion of statistical analysis. The authors declare no conflict of interest.

\section{References}

1. Kumar A, Roberts D, Wood KE, Light B, Parrillo JE, Sharma S, et al. Duration of hypotension prior to initiation of effective antimicrobial therapy is the critical determinant of survival in human septic shock. Crit Care Med. 2006; 34:1589-96.

2. Dellinger RP, Levy MM, Carlet JM, Surviving Sepsis Campaign Management Guidelines Committee. Surviving sepsis campaign: international guidelines for management of severe sepsis and septic shock: 2008. Crit Care Med. 2008; 36:296-327.

3. Colodner R, Rock W, Chazan B, Keller N, Guy N, Sakran W, et al. Risk factors for the development of extended-spectrum beta-lactamase-producing bacteria in nonhospitalized patients. Eur J ClinMicrobiol Infect Dis. 2004; 23:163-7.

4. Rodr guez-Ba oJ,Alcal JC, Cisneros JM, Grill F, Oliver A, Horcajad JP, et al. Community infections caused by extended-spectrum beta-lactamase-producing Escherichia coli. Arch Intern Med. 2008;168:1897-902.

5. Mirelis B, Navarro F, Miro E, Mesa RJ, Coll P, Prats G. Community transmission of extended-spectrum betalactamase. Emerg Infect Dis. 2003; 9:1024-5.

6. Yilmaz E, Akalin H, Ozbey S, Kordan Y, Sinirta M, G rc ogluE, et al. Risk factors in community-acquired/ onset urinary tract infections due to extended-spectrum beta-lactamase-producing Escherichia coli and Klebsiella pneumoniae. J Chemother. 2008; 20:581-5.

7. Ling TK, Xiong J, Yu Y, Lee CC, Ye H, Hawkey PM. Multicenter antimicrobial susceptibility survey of 
gram-negative bacteria isolated from patients with community-acquired infections in the People's Republic of China. Antimicrob Agents Chemother. 2006; 50:41-67.

8. Kang CI, Cheong HS, Chung DR, Peck KR, Song JH, Oh MD, et al. Clinical features and outcome of community-onset bloodstream infections caused by extended-spectrum beta-lactamase-producing Escherichia coli. Eur J Clin Microbiol Infect Dis. 2008; 27:85-8

9. Apisarnthanarak A, Kiratisin P, Saifon P, Kitphati R, Dejsirilert S, Mundy LM. Clinical and molecular epidemiology of community-onset, extended-spectrum beta-lactamase-producing Escherichia coli infections in Thailand: a case-case-control study. Am J Infect Control. 2007; 35:606-12

10. Kollef MH. Inadequate antimicrobial treatment: an important determinant of outcome for hospitalized patients. Clin Infect Dis. 2000; 31(Suppl 4):S131-8

11. Ibrahim EH, Sherman G, Ward S, Fraser VJ, Kollef MH. The influence of inadequate antimicrobial treatment of bloodstream infections on patient outcome in the ICU setting. Chest. 2000; 118:146-55.

12. Melzer M, Petersen I. Mortality following bacteraemic infection caused by extended spectrum beta-lactamase (ESBL) producing E. coli compared to non-ESBL producing E. coli. J Infect. 2007; 55:254-9.

13. Lautenbach E, Patel JB, Bilker WB, Edelstein PH, Fishman NO. Extended-spectrum beta-lactamaseproducing Escherichia coli and Klebsiella pneumoniae: risk factors for infection and impact of resistance on outcomes. Clin Infect Dis. 2001; 32:1162-71.

14. Sobel JD, Kaye D. Urinary tract infections. In: Mandell GL, Bennett JE, Dolin R, editors. Mandell, Douglas, and Bennett's Principles and Practice of Infectious Diseases. 7th ed. Philadelphia:Churchill Livingston; 2009, p. 957-85.

15. Donowitz GR. Pleuropulmonary and bronchial infections. In: Mandell GL, Bennett JE, Dolin R, editors. Mandell, Douglas, and Bennett's Principles and Practice of Infectious Diseases. 7th ed. Philadelphia: Churchill Livingston; 2009, p. 891-916.

16. Sifri $\mathrm{CD}$, Madoff LC. Intra-abdominal infections. In: Mandell GL, Bennett JE, Dolin R, editors. Mandell, Douglas, and Bennett's Principles and Practice of Infectious Diseases. 7th ed. Philadelphia:Churchill Livingston; 2009, p. 1035-44.

17. Pasternack MS, Swartz MN. Skin and soft tissue infections. In: Mandell GL, Bennett JE, Dolin R, editors. Mandell, Douglas, and Bennett's Principles and
Practice of Infectious Diseases. 7th ed. Philadelphia: Churchill Livingston; 2009, p. 1289-312.

18. CLSI (NCCLS). Performance standards for antimicrobial susceptibility testing; eighteenth informational supplement, M100-S18. Vol. 28. Wayne:Clinical Laboratory Standards Institute; 2008.

19. Sun GW, Shook TL, Kay GL. Inappropriate use of bivariable analysis to screen risk factors for use in multivariable analysis. J Clin Epidemiol. 1996; 48: 907-16.

20. Chayakulkeeree M, Junsriwong P, Keerasuntonpong A, Tribuddharat C, Thamlikitkul V. Epidemiology of extended-spectrum beta-lactamaseproducing gramnegative bacilli at Siriraj Hospital, Thailand, 2003. Southeast Asian J Trop Med Public Health. 2005; 36: 1503-9.

21. Apisarnthanarak A, Kiratisin P, Saifon P, Kitphati R, Dejsirilert S, Mundy LM. Predictors of mortality among patients with community-onset infection due to extended-spectrum beta-lactamase-producing Escherichia coli in Thailand. Infect Control Hosp Epidemiol. 2008;29:80-2.

22. Paterson DL, Ko WC, Von Gottberg A, Mohapatra S, Casellas JM, Goossens H, et al. Antibiotic therapy for Klebsiella pneumoniae bacteremia: implications of production of extended-spectrum beta-lactamases. Clin Infect Dis. 2004;39:31-7.

23. Ben-Ami R, Rodr guez-Ba o J, Arslan H, Pitout JD, Quentn C, Calbo ES, et al. A multinational survey of risk factors for infection with extended-spectrum beta-lactamase-producing enterobacteriaceae in nonhospitalized patients. Clin Infect Dis. 2009;49: 682-90.

24. Calbo E, Roman V, Xercavins M, G mez L, Vidal CG, Quintana S, et al. Risk factors for community-onset urinary tract infections due to Escherichia coli harbouring extended-spectrum beta-lactamases. J Antimicrob Chemother. 2006; 57:780-3.

25. Mart nezJA, Aguilar J, Almela M, Marco F, Soriano A, L pez F, et al. Prior use of carbapenems may be a significant risk factor for extended-spectrum betalactamase-producing Escherichia coli or Klebsiella spp. in patients with bacteraemia. J Antimicrob Chemother. 2006;58:1082-5.

26. Friedman ND, Kaye KS, Stout JE, McGarry SA, Trivette SL, Briggs JP, et al. Health care-associated bloodstream infections in adults: a reason to change the accepted definition of community-acquired infections. Ann Intern Med. 2002;137:791-7.

27. Rodriguez-Bano J, Picon E, Gijon P, Hernandez JR, 
Ru z M, Pe a C, et al. Spanish network for research in infectious diseases (REIPI). Community-onset bacteremia due to extended-spectrum beta-lactamaseproducing Escherichia coli: risk factors and prognosis. Clin Infect Dis. 2010; 50:40-8.

28. Harris AD, Karchmer TB, Carmeli Y, Samore MH. Methodological principles of case-control studies that analyzed risk factors for antibiotic resistance: a systematic review. Clin Infect Dis. 2001; 32:1055-61.

29. Harris AD, Samore MH, Lipsitch M, Kaye KS, Perencevich E, Carmeli Y. Control-group selection importance in studies of antimicrobial resistance: examples applied to Pseudomonas aeruginosa, Enterococci, and Escherichia coli. Clin Infect Dis. 2002; 34:1558-63.

30. Behar PR, Teixeira PJ, Fachel JM, Kalil AC. The effect of control group selection in the analysis of risk factors for extended spectrum beta-lactamaseproducing Klebsiella pneumoniae infections. A prospective controlled study. J Hosp Infect. 2008; 68: 123-9.

31. Warren JW, Abrutyn E, Hebel JR, Johnson JR, Schaeffer AJ, Stamm WE. Guidelines for antimicrobial treatment of uncomplicated acute bacterialcystitis and acute pyelonephritis in women. Infectious Diseases Society of America (IDSA). Clin Infect Dis. 1999; 29:745-58.

32. Dow G, Rao P, Harding G, Brunka J, Kennedy J, Alfa M, et al. A prospective, randomized trial of 3 or 14 days of ciprofloxacin treatment for acute urinary tract infection in patients with spinal cord injury. Clin Infect Dis. 2004; 39:658-64.

33. Paterson DL, Mulazimoglu L, Casellas JM, Ko WC, Goossens H, Von Gottberg A, et al. Epidemiology of ciprofloxacin resistance and its relationship to extended-spectrum beta-lactamase production in Klebsiellapneumoniae isolates causing bacteremia. Clin Infect Dis. 2000; 30:473-8.

34. Oktem IM, Gulay Z, Bicmen M, Gur D. HITIT Project Study Group. qnrA prevalence in extended-spectrum beta-lactamase-positive Enterobacteriaceae isolates from Turkey. Jpn J Infect Dis. 2008; 61:13-7.

35. LavillaS, Gonzalez-Lopez JJ, Sabate M, GarciaFernandez A, Larrosa MN, Bartolome RM, et al. Prevalence of qnr genes among extended-spectrum beta-lactamase-producing enterobacterial isolates in Barcelona, Spain. J Antimicrob Chemother. 2008; 61: 291-5. 